\title{
A New COL3AI Mutation in Ehlers-Danlos Syndrome Vascular Type With Different Phenotypes in the Same Family
}

Vascular and Endovascular Surgery

2017, Vol. $51(3) 141-145$

(C) The Author(s) 2017

Reprints and permission:

sagepub.com/journalsPermissions.nav DOI: I0.1 I77/I5385744|7692|I4

journals.sagepub.com/home/ves

(S)SAGE

\author{
Francesca Cortini, $\mathrm{PhD}^{1,2}$, Barbara Marinelli, PhD', Silvia Romi, PhD ${ }^{1,2}$, \\ Agostino Seresini, PhD ${ }^{2,3}$, Angela Cecilia Pesatori, MD', Manuela Seia, MD², \\ Nicola Montano, MD $^{4}$, and Alessandra Bassotti, MD $^{5}$
}

\begin{abstract}
Vascular Ehlers-Danlos syndrome (vEDS) is a rare and severe connective tissue disorder caused by mutations in the collagen type III alpha I chain (COL3AI) gene. We describe a pathogenetic heterozygous COL3AI mutation c.3I40 G>A, P. GlyI047Asp, identified using next-generation sequencing, in a 40-year-old Italian female. The genetic test performed on her relatives, which present different clinical phenotypes, confirmed that they carry the same mutation in heterozygous state. This finding confirms that mutations causing vEDS have an incomplete penetrance.
\end{abstract}

\section{Keywords}

Ehlers-Danlos syndrome vascular type, COL3Al gene, next-generation sequencing, Haloplex target enrichment, penetrance, bioinformatics analysis

\section{Introduction}

Vascular Ehlers-Danlos syndrome (vEDS) is a heritable connective tissue disorder mainly characterized by severe arterial and gastrointestinal complications. ${ }^{1}$ The clinical diagnosis of vEDS can be suspected on the basis of criteria established in $1997^{2}$ : a characteristic facial aspect (acrogeria); thin and translucent skin with highly visible subcutaneous blood vessels; ecchymoses and hematomas; and arterial, digestive, and obstetrical complications. However, in most individuals, the diagnosis, in the absence of a family history of the disorder, is not suspected until arterial aneurysm and/or dissection, bowel perforation, or organ rupture occur. Therefore, in the presence of these complications, the genetic analysis of collagen type III alpha I chain $(C O L 3 A 1)$ gene is necessary to confirm the diagnosis. ${ }^{3}$

COL3A1, encoding type III collagen, is situated on the long arm of chromosome 2 in position 2q24.3-q31 (Figure 1). Vascular Ehlers-Danlos syndrome is caused by a deficit of type III collagen, which belongs to the fibrillar collagens. All fibrillar collagens are homo-/heterotrimers formed by $3 \alpha$-chains. Type III collagen is a homotrimer formed by the binding of $3 \alpha 1$ (III) chains; the central region of each chain adopts a triple-helix structure. The amino acid sequence of the triple helix is characterized by repetition of Gly-X-Y sequences, where $\mathrm{X}$ and $\mathrm{Y}$ are other amino acids (often proline and hydroxyproline, respectively), in order to maintain a correct binding of $\alpha$-monomers (Figure 1). Type III collagen is a fundamental element of arterial vessels and walls of gastrointestinal tracts. COL3A1's product quantitative or qualitative deficit in patients with vEDS increases the possibility of occurrence of arterial tears or dissections, peculiar tracts of this pathology.

In the present study, we analyzed genotype/phenotype correlation of a COL3A1 mutation c. $3140 \mathrm{G}>\mathrm{A}$, p. Gly1047Asp ${ }^{4}$ identified in an Italian family affected by vEDS. The clinical and genetic counseling are carried out at the Regional Center of Ehlers-Danlos Syndrome (Ospedale Maggiore Policlinico, Milan, Italy) where the proband, a 40-year-old Italian female, and her relatives have been analyzed (Figure 2). They present different clinical phenotypes confirming a different penetrance occurring in different members of the same family.

'Department of Clinical Sciences and Community Health, University of Milan IRCCS Ca' Granda Foundation, Milano, Italy

${ }^{2}$ Genetics Laboratory, IRCCS Ca' Granda Foundation, Milan, Italy

${ }^{3}$ Fondazione Grigioni per il Morbo di Parkinson, Milano, Italy

${ }^{4}$ Department of Internal Medicine, Fondazione IRCCS Ca' Granda-Ospedale

Maggiore \& Department of Clinical Sciences and Health Community, University of Milan, Italy

${ }^{5}$ Regional Center of Ehlers-Danlos Syndrome, IRCCS Ca' Granda Foundation, Milan, Italy

\section{Corresponding Author:}

Francesca Cortini, Department of Clinical Sciences and Community Health, University of Milan, Via San Barnaba 8, Milan 20122, Italy.

Email: francesca.cortini@guest.unimi.it 


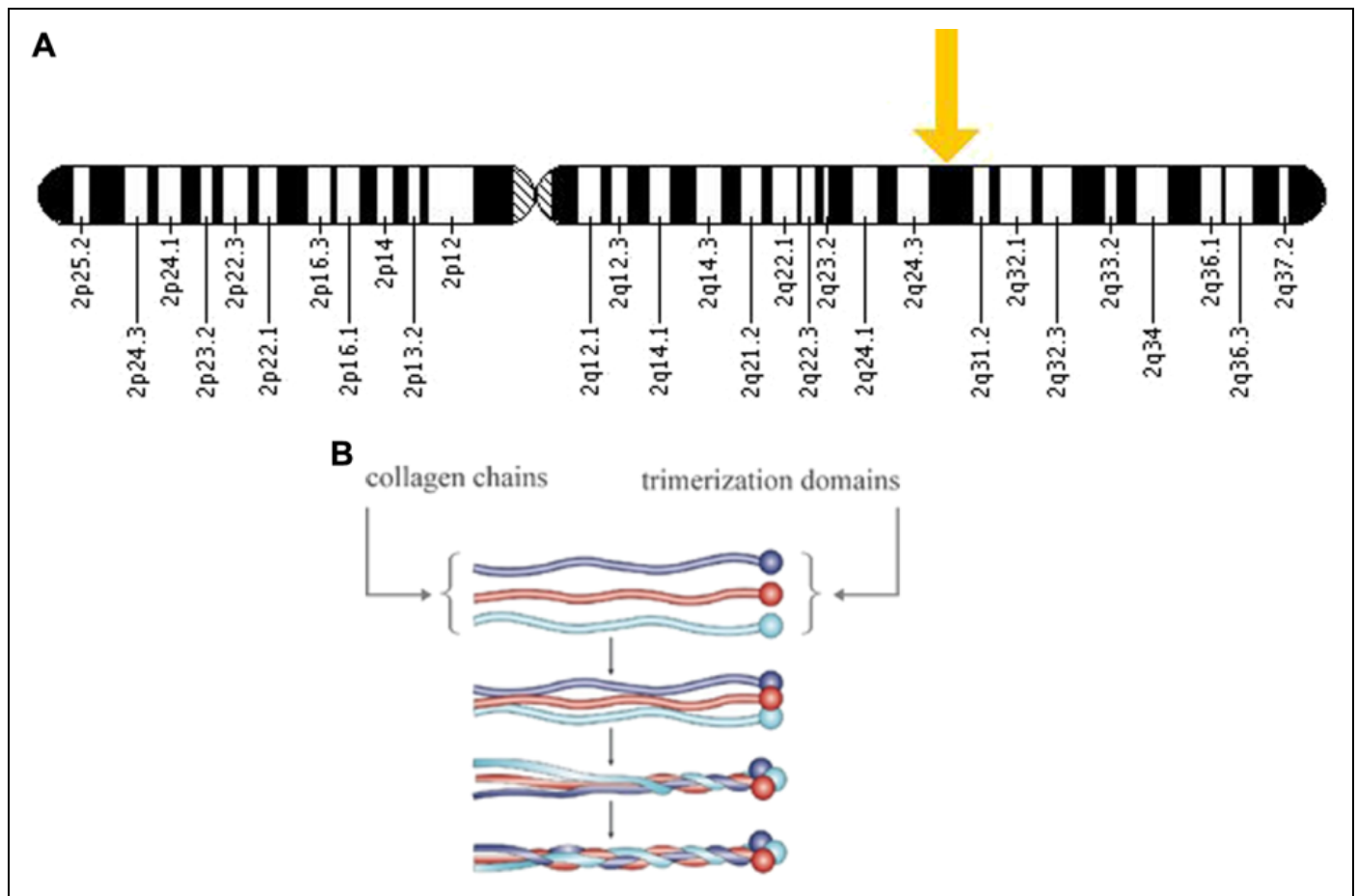

Figure I. COL3AI Gene. A, Yellow arrow indicates the location of COL3AI gene. B, Schematic representation of collagen fiber; the COL3AI protein structure is homotrimer formed by $3 \alpha \mathrm{I}$ (III) chains.

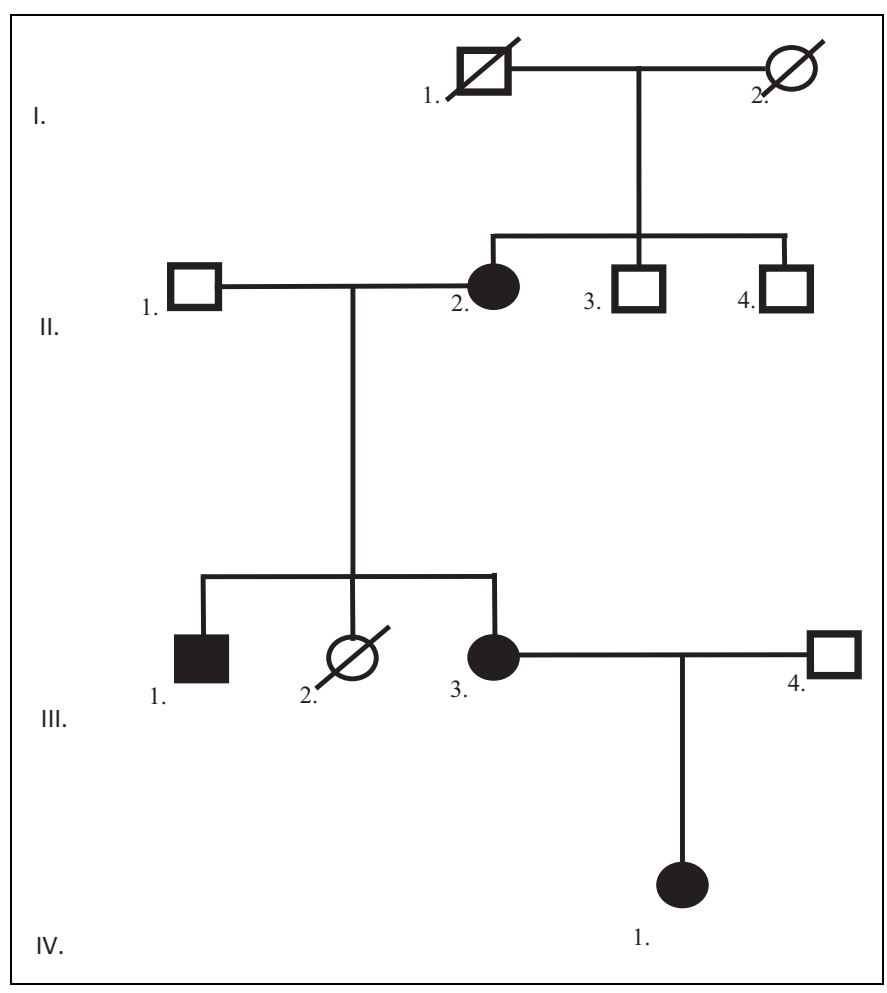

Figure 2. Pedigree. In black member of the family heterozygous of the c.3 I40G>A (p.Gly 1047Asp) mutation in exon 44 of COL3AI gene. I:I and $\mathrm{l}: 2$ were died at 70 and 80 years for lung tumor and ictus, respectively.

\section{Material and Methods}

\section{Patients}

A 40-year-old Italian female (III:3, Figure 2) was admitted to the Regional Center of Ehlers-Danlos Syndrome (Ospedale Maggiore Policlinico, Milan, Italy) for a hemorrhagic episode with partial separation of the placenta in the seventh week of gestation. In 2012, after a second abortion, she underwent 3 surgeries for uterus recanalization. After that, she did not refer any problems. The clinical history revealed smooth, velvet, and thin skin, slightly elastic in correspondence of forearms and extremely translucent with visible veins in correspondence of chest and abdomen, and acrogeria of the hands and feet (Figure 3). She also referred capillary fragility at the lower limbs - she underwent sclerotherapy. She had a baby (IV:1, Figure 2) that was born after 30th week of gestation and presented lung problems.

At the same time, other members of her family were clinically diagnosed at the Regional Center of Ehlers-Danlos Syndrome. Mother's clinical spectrum (II:2, Figure 2) didn't reveal typical vEDS characteristics. In 2012, she had a vascular episode of aneurysm of hepatic artery that arose the suspect for vEDS. Brother's clinical spectrum (III:1, Figure 2) shows slightly elastic skin in correspondence of knees, elbows, and hands; visible veins on chest and abdomen; and atrophic scars. Whereas the sister (III:2, Figure 2) died when she was a 7-month-old baby. Proband's daughter (IV:1, Figure 2) had lung problems, but other clinical examinations will be 


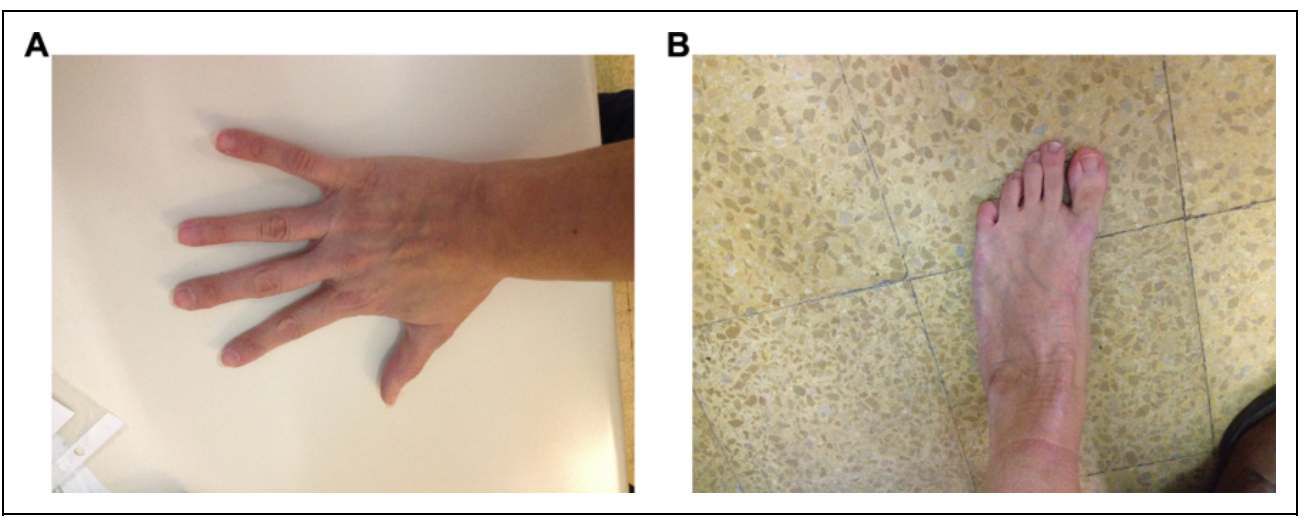

Figure 3. Acrogeria. A, Particular of hand. B, Particular of foot of a proband.

Table I. NGS Panel: The Genes Included in the NGS Panel Are Clinically Implicated in EDS.

\begin{tabular}{lll}
\hline Gene & Phenotype & Chromosome \\
\hline ADAMTS2 & EDS type VIIC & chr5q.35.3 \\
B4GALT7 & EDS progeroid type I & chr5q35.3 \\
CHSTI4 & EDS musculocontractural type I & chrl5qI5.I \\
COL3AI & EDS type IV & chr2q32.2 \\
COL5AI & EDS classic type & chr9q34.3 \\
COL5A2 & EDS classic type & chr2q32.2 \\
PLODI & EDS type VI & chrlp36.22 \\
PLOD3 & Lysyl hydroxylase 3 deficiency & chr7q22.I \\
TNXB & EDS due to tenascin X deficiency & chr6p2I.33 \\
\hline
\end{tabular}

Abbreviations: ADAMTS2, ADAM Metallopeptidase With Thrombospondin Type I Motif 2; B4GALT7, Beta-I, 4-Galactosyltransferase 7; CHST I4, Carbohydrate Sulfotransferase I4; COL3AI, collagen type III alpha I chain; COL5AI, collagen type $\mathrm{V}$ alpha I chain; COL5A2, collagen type $\mathrm{V}$ alpha II chain; EDS, Ehlers-Danlos syndrome; PLODI, Procollagen-Lysine,2-Oxoglutarate 5-Dioxygenase I; PLOD3, Procollagen-Lysine,2-Oxoglutarate 5-Dioxygenase 3; TNXB, Tenascin XB.

necessary to fully understand her phenotype. The remaining members of the family (II:3, II.4, III:4; Figure 2) were clinically evaluated but they did not show relevant clinical issues. We didn't perform genetic test on I:1 and I:2 (Figure 2) because they died for lung tumor and ictus at 70 and 80 years, respectively, before this clinical investigation.

\section{Haloplex Target Enrichment}

We adopted next-generation sequencing (NGS) for investigating genomic regions of interest through target enrichment. It was performed by means of Haloplex Target Enrichment kit (Agilent Technologies, CA, USA). Probes for all coding exons, including intron-exon boundaries, were designed by the Haloplex SureDesign website (www.genomics.agilent.com). The 9 genes included in the design were selected on the basis of their clinical characteristics (www.le.ac.uk/ge/collagen; Table 1). The total region size was 100.336 kilo base pair $(\mathrm{kbp})$ for an actual analyzed target of $99.718 \mathrm{kbp}$ bases. Enrichment was performed according to supplier's protocol (version D.6, August
2014). A total of $225 \mathrm{ng}$ DNA was digested in 8 different restriction reactions during 30 minutes at $37^{\circ} \mathrm{C}$. The 8 reaction digestions were combined into a single hybridization mix containing target-specific probes. Hybridization reaction was performed in 3-hour reaction at $54^{\circ} \mathrm{C}$. Probes were labeled with biotin and designed to hybridize to both ends of the digested fragments, therefore generating circular fragments containing 1 nick. Then, the DNA probe hybrids were captured with streptavidin beads to eliminate linear, nontarget DNA fragments. In a second ligation reaction, the remaining nick was closed to complete circularization. DNA captured was eluted from the beads and amplified by polymerase chain reaction, followed by a final purification reaction with AMPure XP beads (Beckman Coulter, Fullerton, California).

Finally, the concentration of each library was measured using Tape Station software A.02.01 (SR1), KGGSeq software 3.0 and after different elution with Tris Edta (TE) arrived at $4 \mathrm{nmol}$. Then the final pool was obtained by mixing different genomic libraries, and the ideal final concentration is $8 \mathrm{pmol}$ (ideal concentration for cluster density about 900/1000 $\mathrm{K} / \mathrm{mm}^{2}$ ). Captured genomic libraries were sequenced to generate $2 \times 150$ bp paired end reads using the Illumina MiSeq, CA, USA.

\section{Next-Generation Sequencing Data Analysis}

To analyze the generated FastQ files (file format used to store biological sequence and its quality score), a workflow was set up using Galaxy software (https://orione.crs4.it/). ${ }^{5}$ FastQ files were evaluated by quality and trimming, aligned, and mapped to the human reference genome (GRCh37/hg19) using Burrows-Wheeler Aligner. ${ }^{6,7}$ Alignments including sorting, merging, indexing, and generating alignments in a perposition format were manipulated by SAM tools ${ }^{8,9}$ to generate BAM (binary format for storing sequence) file. Local realignment, base quality recalibration, and variant calling were performed using Genome Analysis Toolkit version 2.0 (Broad Institute, Cambridge, Massachusetts), ${ }^{10}$ applying this quality parameters - coverage $>20$, base quality score $>30$, and 


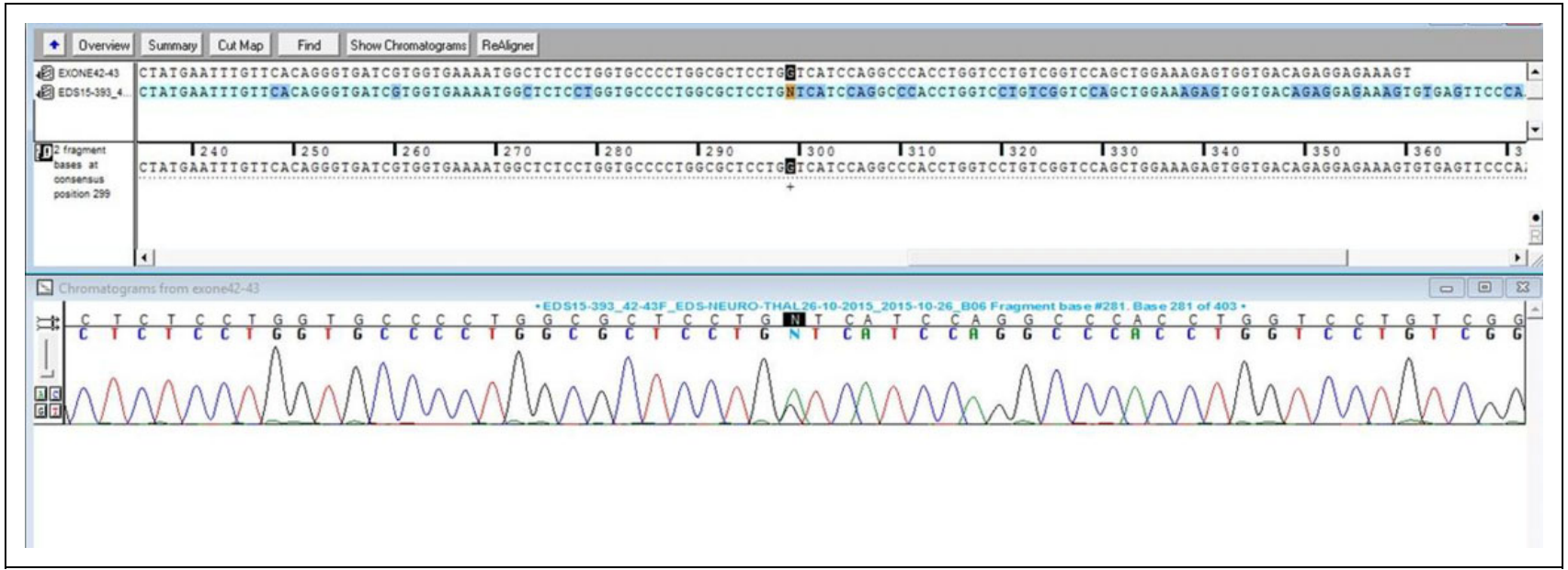

Figure 4. Electropherogram. Missense mutation c.3 I40G>A, p.Gly I047Asp identified in exon 44 of COL3AI gene.

mapping quality $>20$. Variants that did not pass these quality values were removed.

Finally, the variant calling files were filtered through different parameters as genotype quality filters, gene feature filters (missense, splicing, frameshift), and functional impact filters using KGGSeq software. ${ }^{11}$

\section{Sanger Sequencing}

The variants found with NGS target enrichment method were confirmed by Sanger method using Big Dye Terminator Cycle Sequencing kit (Applied Biosystems, Life Technologies, CA, USA), followed by capillary electrophoresis on an ABI3130xl (Applied Biosystems). The electropherograms were analyzed by Sequencer software v5.1.

\section{Data Analysis of Missense Variants}

To investigate the pathogenetic properties of missense variants, different software were used-SIFT/PROVEAN, Polyphen-2, Mutation Taster, Grantham Distance, and Align GVGD, which were based on sequence homology, the physiochemical similarity between the alternate amino acids, effect on an amino acid substitution on the structure, and function of a protein and conservation level of the amino acid residue among species, respectively. ${ }^{12,13}$

\section{Results}

Genetic analysis done on the proband (III:3; Figure 2) by NGS target enrichment approach identified a mutation in exon 44 of COL3A1 gene: c.3140G $>$ A, p. Gly1047Asp in heterozygous state (Figure 4$)^{4}$ (www.le.ac.uk/ge/collagen/), confirmed by Sanger method on a new DNA extraction. The clinical counseling on mother, brother, and daughter (II:2, III:1, IV:1; Figure 2) and genetic analysis, by Sanger method, revealed that they were carrier of the same mutation in heterozygous state, whereas the remaining members of the family were found negative (II:3, II:4, III:4; Figure 2).

The c.3140G $>$ A, p.Gly1047Asp mutation is a missense variant that leads to the substitution of a neutral amino acid, the glycine, with a polar one, the aspartic acid. It is located in the triple-helix region of the protein. As previously described, type III collagen consists of repetition of Gly-X-Y, the peculiar feature of the $3 \alpha$-chains that forms collagen type III. The substitution of the first glycine, a neutral amino acid, with a polar amino acid modifies the characteristic $\alpha$-helix structure, and this causes qualitative and quantitative defects of mature type III collagen. ${ }^{14}$ The pathogenicity features of this substitution has been confirmed by bioinformatic analyses. In particular, SIFT, PROVEAN, Polyphen-2, Mutation Taster, Grantham Distance, and Align GVGD ${ }^{12,13}$ indicate that p.Gly1047Asp substitution is damaging and alters the structure of the protein.

Moreover, the missense variant didn't explain the different phenotypes of other family members (Figure 2). ${ }^{15}$ The proband (III:3, Figure 2) shows the main characteristics of vEDS, whereas mother and brother showed isolate vascular episodes; up to date the daughter is under analysis. The phenotypical variability of affected members within the same family confirmed that genetic testing is essential to confirm the diagnosis of vEDS.

\section{Discussion}

In this study, we described a COL3A1 missense mutation c.3140G $>$ A, p.Gly1047Asp identified in an Italian family (Figure 4). It is a substitution of a glycine, neutral amino acid, with an aspartic acid, polar amino acid, located in triple-helix region of the protein structure. Bioinformatic analysis ${ }^{12,13,16,17}$ confirmed its pathogenic properties. The clinical counseling revealed a different penetrance occurring in the same family. ${ }^{15}$

In fact, the proband (III:3, Figure 2) had the main characteristics of vEDS - smooth, velvet, and thin skin with visible veins in correspondence of abdomen and chest and acrogeria at the feet and hands. She shows fragility of veins, capillaries, and 
tissues; in fact, she needed to undergo 3 surgeries to achieve uterus recanalization after the second abortion. Her mother (II:2) and her brother (III:1) had isolated vascular episode; the clinical and genetic diagnoses of the proband suggest the possibility of familiar vEDS that was confirmed by genetic testing of COL $3 A 1$ performed on the other members of the family by Sanger sequencing.

At the time of the first counseling, the proband was pregnant and she had the baby at the 30th week of gestation. She was convinced by her clinical and genetic data to try genetic test on her baby. The daughter resulted to be carrier of the same mutation in heterozygous state, but the clinical phenotype is incomplete for the early age of the baby. The other members of the family were found negative (Figure 2).

The affected members of family follow a treatment with celiprorol, a cardioselective $\beta$-blocker with $\beta_{2}$ antagonist vasodilatatory properties. Celiprorol was reported to reduce heart rate and pulsatile pressures in essential hypertension ${ }^{15}$ and could decrease the continuous and pulsatile mechanical stress on collagen fibers within the arterial wall.

In conclusion, c. $3140 \mathrm{G}>\mathrm{A}$, p. Gly1047 $\mathrm{Asp}^{4}$ is a pathogenetic mutation. Our analysis shows that this mutation influences the phenotype with different penetrance, as demonstrated by different disease manifestations in different members of the same family. ${ }^{18}$

We hypothesize that the heterozygous state of the mutation is the possible determinant of different phenotypes observed. In any case, we cannot exclude other genetic factors influencing the expression of this mutation in this family. Ultimately, this study confirmed that the genetic test is essential to evaluate the nature and the risks of the complications in patients with vEDS.

\section{Declaration of Conflicting Interests}

The author(s) declared no potential conflicts of interest with respect to the research, authorship, and/or publication of this article.

\section{Funding}

The author(s) received no financial support for the research, authorship, and/or publication of this article.

\section{References}

1. Pepin M, Schwarze U, Superti-Furga A, Byers PH. Clinical and genetic features of Ehlers-Danlos syndrome type IV, the vascular type. N Engl J Med. 2000;342(10):673-680.

2. Beighton P, De Paepe A, Steinmann B, Tsipouras P, Wenstrup RJ. Ehlers-Danlos syndromes: revised nosology, Villefranche, 1997. Ehlers-Danlos National Foundation (USA) and Ehlers-Danlos Support Group (UK). Am J Med Genet. 1998;77(1):31-37.

3. Superti-Furga A, Gugler E, Gitzelmann R, Steimann B. EhlersDanlos syndrome type IV: a multi-exon deletion in one of the two
COL3A1 alleles affecting structure, stability, and processing of type III procollagen. J Biol Chem. 1988;263(13):6226-6232.

4. Pepin MG, Schwarze U, Rice KM, Liu M, Leistritz D, Byers PH. Survival is affected by mutation type and molecular mechanism in vascular Ehlers-Danlos syndrome (EDS type IV). Genet Med. 2014;16(12):881-888.

5. Cuccuru G, Orsini M, Pinn A, et al. Orione, a web-based framework for NGS analysis in microbiology. Bioinformatics. 2014; 30(13):1928-1929.

6. Li H, Durbin R. Fast and accurate short read alignment with Burrows-Wheeler transform. Bioinformatics. 2009;25(14): 1754-1760.

7. Li H, Durbin R. Fast and accurate long-read alignment with Burrows-Wheeler transform. Bioinformatics. 2010;26(5):589-595.

8. Li H, Handsaker B, Wysoker A, et al; 1000 Genome Project Data Processing Subgroup. The Sequence Alignment/Map format and SAMtools. Bioinformatics. 2009;25(16):2078-2079.

9. Li H. A statistical framework for SNP calling, mutation discovery, association mapping and population genetical parameter estimation from sequencing data. Bioinformatics. 2011;27(21):2987-2993.

10. McKenna A, Hanna M, Banks E, et al. The Genome Analysis Toolkit: a MapReduce framework for analyzing next-generation DNA sequencing data. Genome Res. 2010;20(9):1297-1303.

11. Li MX, Gui HS, Kwan JS, Bao SY, Sham PC. A comprehensive framework for prioritizing variants in exome sequencing studies of Mendelian diseases. Nucleic Acids Res. 2012;40(7): e53.

12. Adzhubei I, Jordan MD, Sunyaev SR. Predicting functional effect of human missense mutations using PolyPhen-2. Curr Protoc Hum Genet. 2013; Chapter 7: Unit 7.20.

13. Kumar P, Henikoff S, Ng PC. Predicting the effects of coding non-synonymous variants on protein function using the SIFT algorithm. Nat Protoc. 2009;4(7):1073-1081.

14. Tavtigian SV, Deffenbaugh AM, Yin L, et al. Comprehensive statistical study of 452 BRCA1 missense substitutions with classification of eight recurrent substitutions as neutral. J Med Genet. 2006;43(4):295-305.

15. Choi Y, Sims GE, Murphy S, Miller JR, Chan AP. Predicting the functional effect of amino acid substitutions and indels. PLoS One. 2012;7(10): e46688.

16. Watanabe A, Takashi S. The vascular type of Ehlers-Danlos syndrome. J Nippon Med Sch. 2008;75(5):254-261.

17. Boutouyrie P, Bussy C, Hayoz D, et al. Local pulse pressure and regression of arterial wall hypertrophy during long-term antihypertensive treatment. Circulation. 2000;101(22): 2601-2606.

18. Kuivaniemi H, Tromp G, Prockop DJ. Mutations in fibrillary collagens (types I, II, III and XI), fibril-associated collagen (type IX), and network forming collagen (type X) cause a spectrum of disease bone, cartilage, and blood vessels. Hum Mutat. 1997;9(4): 300-315. 